\title{
Revealing the Correlation of the Electrochemical Properties and the Hydration of Inkjet-Printed CdSe/CdS Semiconductor Gels
}

\author{
Jan F. Miethe, Franziska Luebkemann, Anja Schlosser, Dirk Dorfs, and Nadja C. Bigall*
}

Cite This: https://dx.doi.org/10.1021/acs.langmuir.9b03708

Read Online

ABSTRACT: The mobility of charge carriers across a semiconductor-nanoparticle-based 3D network (i.e., a gel) and the interfacial transfer of the charge carriers across the nanoparticle network/electrolyte boundary are elementary processes for applications in the fields of sensing and energy harvesting. The automated manufacturing of electrodes coated with porous networks can be realized by inkjet printing. By simultaneous printing of $\mathrm{CdSe} / \mathrm{CdS}$ dot-in-rodshaped nanorods (NRs) and the destabilization reagent, $\mathrm{CdSe} / \mathrm{CdS}$ gel-networkcoated electrodes can be obtained. In this work, the charge carrier mobility of the electrons and the holes within the porous $\mathrm{CdSe} / \mathrm{CdS}$ nanorod gel network is investigated via photoelectrochemistry. Using linear sweep voltammograms

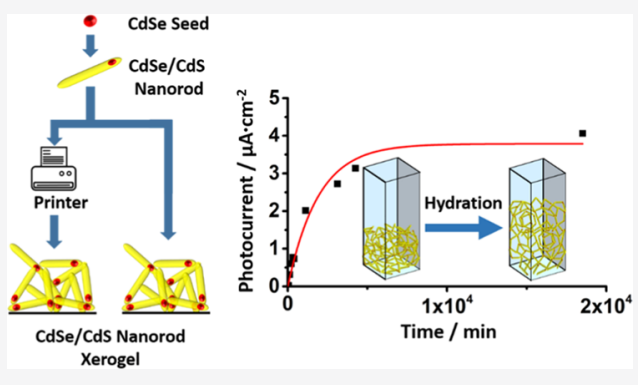
(LSVs) and intensity-modulated photocurrent spectroscopy (IMPS), it is shown that the electron is moving within the tip-to-tip-connected CdSe/CdS NR gel structure, while the holes are trapped in the CdSe seed of the semiconductor heterostructures. Furthermore, the preparation process of gel structures is related to the elementary mechanism of hydration, which can be shown via photoelectrochemical long-term studies.

\section{INTRODUCTION}

One elementary challenge of the technical and economical mastering of fields like photoelectrochemical sensing and energy harvesting is the realization of interfaces that present a high specific surface and electronic conductivity. Since the desired systems have to be addressable by light, the implementation of suitable porous semiconductor networks as chemical-optic-electronic cross sections can be considered as the main step in the realization of the above-mentioned applications. For building such networks, a good control over the nanocrystal building blocks, which in the next step are assembled, is necessary. Today, a significant number of preparative ways to yield II-VI semiconductor nanocrystals, e.g., CdTe, CdSe, CdS, and $\mathrm{ZnS},{ }^{1-5}$ and III-V semiconductor nanocrystals, e.g., InP, GaAs, and $\mathrm{GaP}^{6-9}$ are well described in the literature. A further step in the evolution of nanochemistry is the development of heteronanoparticles, which are composites of different semiconductor materials with various band-gap sizes. One prominent example of previously investigated heterostructures is $\mathrm{CdSe} / \mathrm{CdS} .^{10,11}$ This material combination leads to a pseudo-type-II heterostructure, as the conduction bands of both semiconductors are nearly aligned. In contrast to this, the CdSe valence band has a significantly higher energetic position than the valence band of CdS. Due to this alignment, the photoexcited electron in $\mathrm{CdSe} / \mathrm{CdS}$ core/ shell heterostructures is delocalized over the whole particle, while the photoexcited hole is fixed in the CdSe core. The general term to summarize different chemical and physical ways to generate voluminous and porous solid-state networks is gelation. ${ }^{12}$ While all mentioned particles can be applied as building blocks for the preparation of macroscopic gel networks, a network of pseudo-type-II heterostructures is expected to enable the selective mobility of one part of the exciton and is therefore of particular interest. ${ }^{13,14}$ For nanocrystal gelation, in general, there are several synthetic routes, which have already been mentioned in the literature. Gelation is usually achieved via controlled destabilization of a solution of the colloidal nanoparticles. The most common way of destabilization is induced via a destabilization agent, which detaches stabilizing ligands from the surface of the particles. $^{15-18}$ Other common ways to obtain gels are, among others, the fast freezing of particle solutions and the gelation via photoexcitation. ${ }^{19-21}$

Having in mind the applications mentioned above, we need to develop methods for immobilizing nanocrystal-based gels on conducting electrodes, which was done in recent studies. ${ }^{15,19,22}$ It was possible to develop two distinct ways to prepare CdSe/ $\mathrm{CdS}$ nanorod (NR) gels on electrode surfaces. One method applied to prepare photoelectrodes was to glue vessels made out of poly(methyl methacrylate) (PMMA) cuvettes onto (3mercaptopropyl)trimethoxysilane (MPTMS)-functionalized indium-tin oxide (ITO) slides and to gelate $\mathrm{CdSe} / \mathrm{CdS}$

Received: December 3, 2019

Revised: $\quad$ March 2, 2020

Published: March 3, 2020 
nanorods in these cuvettes. To the best of our knowledge, this is the first publication that mentions this technique to develop nanostructure-covered electrodes. This technique was easy to realize but showed typical disadvantages of a manual preparation, like deficits in the scalability and parameters of gelation, which are complicated to adjust. A more sophisticated manufacturing technique to obtain nanoparticle-based gel networks is based on our previous work about inkjet printing. ${ }^{15}$ The development of nanoparticle-based inks is a promising approach in the direction of technical automation. ${ }^{23-25}$ Using a commercial office printer, we were able to print aqueous solutions of $\mathrm{CdSe} / \mathrm{CdS} \mathrm{NR}$ building blocks and the destabilization agent hydrogen peroxide simultaneously to obtain a printed nanoparticle-based gel-network-coated electrode. ${ }^{23}$ The exact control of the sample dimensions is one benefit of inkjet printing (additionally to others such as the ease of lateral structuration). In this work, one of our main intentions during the development of the presented experimental design was to produce gel electrodes of different thicknesses (5, 25, 100, and 400 printing cycles) and to indepth investigate the influence of the gel thickness on their optoelectrochemical properties. By time-dependent measurements, we were able to investigate the influence of the thickness of the particle network on its kinetics of hydration via external photocurrent efficiency (EPE) spectroscopy, linear sweep voltammograms (LSVs), and intensity-modulated photocurrent spectroscopy (IMPS). IMPS is utilized as one of the most suitable spectroelectrochemical techniques to investigate the process of charge migration through the gel. One experimental benefit of IMPS measurements is the possibility to select the excitation wavelength to obtain a spatial resolution of the gel excitation across its thickness. Using Nyquist plots, we were therewith able to differentiate between the different mechanisms of the charge transport.

\section{EXPERIMENTAL SECTION}

Reagents and Materials. Cadmium oxide (CdO, 99.99\%) and selenium (Se, 99.99\%) were purchased from Alfa Aesar. Tri- $n$ octylphosphine oxide (TOPO, 99\%) and tri- $n$-octylphosphine (TOP, 97\%) were purchased from ABCR. Hexylphosphonic acid (HPA, $\geq 99 \%$ ) and octadecylphosphonic acid (ODPA, $\geq 99 \%$ ) were purchased from PCI synthesis. Hydrogen peroxide $\left(\mathrm{H}_{2} \mathrm{O}_{2}, 35 \%\right)$, nhexane ( $\geq 99 \%)$, 3-mercaptopropionic acid (MPA, $\geq 99 \%)$, (3mercaptopropyl)trimethoxysilane (MPTMS, 95\%), 1-octadecene (ODE, 90\%), sodium sulfite ( $\geq 98 \%$ ), and sulfur (S, 99.98\%) were purchased from Sigma-Aldrich. Methanol $(\geq 99.5 \%)$ and toluene (99.9\%) were purchased from Carl Roth. Tin-doped indium oxide glass (ITO glass), which is based on $1.1 \mathrm{~mm}$ coated unpolished soda lime float glass was purchased from VisionTek and applied as electrode substrate. The substrate shows a surface resistance of 12 ohms/sq. Disposable cuvettes of the type Macro 2.5 to $4.5 \mathrm{~mL}$ (PMMA) were purchased from VWR. A silicon sealing mass of the type Toolcraft Transil Silikon was purchased from Conrad Electronic SE. The inkjet printer model was Epson Expression Premium XP-620.

Synthesis of CdSe Dots. CdSe dots were synthesized using a slightly modified and six times scaled-up procedure developed by Carbone et al. ${ }^{10} \mathrm{CdO}(0.36 \mathrm{~g})$, TOPO (18 g), and ODPA (1.7 g) were placed in a $50 \mathrm{~mL}$ three-neck flask. The reaction mixture was degassed at $150{ }^{\circ} \mathrm{C}$ under vacuum for $1 \mathrm{~h}$. Afterward, the reaction mixture was heated to $300{ }^{\circ} \mathrm{C}$ under an inert gas atmosphere. When the desired temperature was reached, $10.8 \mathrm{~mL}$ of TOP was injected. After a further increase in the reaction temperature to $380{ }^{\circ} \mathrm{C}$, the solution turned clear and a solution of $10.8 \mathrm{~mL}$ of TOP and $4.4 \mathrm{mmol}$ of Se was quickly injected into the reaction mixture. After the recovery of the temperature, the reaction mixture was quenched with ODE. When the reaction mixture was cooled down to $70{ }^{\circ} \mathrm{C}, 25 \mathrm{~mL}$ of toluene was added. CdSe dots were precipitated via the addition of 25 $\mathrm{mL}$ of methanol and centrifuged for $10 \mathrm{~min}$ (3885 RCF). The solution was purified two times by the addition of a 1:1 mixture of toluene/methanol and centrifugation. The CdSe seeds were stored in toluene.

Synthesis of Dot-in-Rod CdSe/CdS NRs. The CdS shell growth is based on the same publication of Carbone et al. as the CdSe seed synthesis. ${ }^{10}$ In an eight times scaled-up synthesis, $0.48 \mathrm{~g}$ of $\mathrm{CdO}, 24 \mathrm{~g}$ of TOPO, $2.32 \mathrm{~g}$ of ODPA, and $0.64 \mathrm{~g}$ of HPA were placed in a 100 $\mathrm{mL}$ three-neck flask and heated up to $150{ }^{\circ} \mathrm{C}$ under vacuum for $1 \mathrm{~h}$. After the reaction mixture was degassed, the reaction temperature was increased to $300{ }^{\circ} \mathrm{C}$ under an argon atmosphere. After reaching this temperature, $12 \mathrm{~mL}$ of TOP was injected, followed by a temperature increase to $380{ }^{\circ} \mathrm{C}$. At this temperature, $2.21 \mathrm{mmol}$ sulfur and $50 \mu \mathrm{M}$ CdSe seeds in $14.4 \mathrm{~mL}$ of TOP were injected into the reaction mixture. The concentration of CdSe seeds was determined by UV/vis spectroscopy, according to the work of $\mathrm{Yu}$ et al. ${ }^{26}$ After the injection, the reaction mixture was further heated for $8 \mathrm{~min}$. The reaction mixture was cooled down to $70^{\circ} \mathrm{C}$ when $25 \mathrm{~mL}$ of toluene was added, and the $\mathrm{CdSe} / \mathrm{CdS}$ NRs were precipitated and cleaned by the same procedure as that for the CdSe seeds.

Phase Transfer of Dot-in-Rod CdSe/CdS NRs. To obtain an aqueous $\mathrm{CdSe} / \mathrm{CdS} \mathrm{NR}$ solution, a phase transfer adapted from Kodanek et al. was performed. ${ }^{27} \mathrm{~A}$ total of $3 \mathrm{~mL}$ of the toluene-based $\mathrm{CdSe} / \mathrm{CdS} \mathrm{NR}$ solution $[c(\mathrm{Cd})=128 \mathrm{mmol} / \mathrm{L}]$ was precipitated with $3 \mathrm{~mL}$ of methanol, centrifuged, and redissolved in $3 \mathrm{~mL}$ of $n$-hexane. ${ }^{27}$ A solution of $10 \mathrm{~mL}$ of methanol, $0.2 \mathrm{~g}$ of $\mathrm{KOH}$, and $0.24 \mathrm{~mL}$ of MPA was added to the CdSe/CdS NR solution, and the mixture was shaken overnight. The methanolic phase was separated and centrifuged. The NRs were stored in $12 \mathrm{~mL}$ of $0.1 \mathrm{M} \mathrm{KOH}$ solution. The cadmium concentration was $32 \mathrm{mmol} / \mathrm{L}$.

Preparation of ITO Substrates. ITO glass slides of approximately $1.5 \times 6 \mathrm{~cm}$ were used as substrates. The slides were cleaned via the Radio Cooperation of America (RCA) process for $2.5 \mathrm{~h}$ at 70 ${ }^{\circ} \mathrm{C}$ with a mixture of $1: 1: 5(\mathrm{v} / \mathrm{v})$ of hydrogen peroxide (35\%), ammonium hydroxide (25\%), and deionized water. ${ }^{28}$ Afterward, the slides were rinsed with water and dried in an airflow. Activated slides were functionalized in a solution of $150 \mathrm{~mL}$ of toluene and $1.5 \mathrm{~mL}$ of (3-mercaptopropyl)trimethoxysilane for $18 \mathrm{~h}$ at $70{ }^{\circ} \mathrm{C}$. Afterward, the slides were rinsed with toluene and dried in an airflow.

Synthesis of CdSe/CdS Gels on ITO Substrates. A disposable UV/vis cuvette, which was made out of PMMA, was cut into two equal pieces and glued onto the conductive side of an MPTMSfunctionalized ITO slide using a silicon sealing mass. An aqueous $\mathrm{CdSe} / \mathrm{CdS} \mathrm{NR}$ solution $(1 \mathrm{~mL})$ was pipetted into the cuvette, and $100 \mu \mathrm{L}$ of a 0.35 wt $\%$ solution of $\mathrm{H}_{2} \mathrm{O}_{2}$ was added. After $3 \mathrm{~h}$, a voluminous precipitate was recognizable, which was cleaned after $24 \mathrm{~h}$ with a fresh $0.1 \mathrm{M} \mathrm{KOH}$ solution and after another $24 \mathrm{~h}$ with deionized water. The excess solution was removed, and the hydrogel was dried under ambient conditions. After three days of drying, a porous nanoparticle gel network was formed and the cuvette was removed carefully.

Inkjet Printing of $\mathrm{CdSe} / \mathrm{CdS}$ Gels on ITO Substrates. Nanoparticle-based xerogel-network-coated electrodes were prepared according to our previous work. ${ }^{15,23}$ The NR ink was filled into the magenta cartridge, and the $0.35 \mathrm{wt} \% \mathrm{H}_{2} \mathrm{O}_{2}$ solution was filled into the cyan cartridge. The printed area of $(0.9 \mathrm{~cm} \times 0.9 \mathrm{~cm})$ was defined in the software CD Print. The ratio of NR solution to destabilization solution was controlled using the CYMK color code. For pure CdSe/ CdS NR films, the printed color was adjusted to $100 \%$ magenta. In the case of $\mathrm{CdSe} / \mathrm{CdS}$ hydrogels, the printed color was programmed to $70 \%$ magenta and $30 \%$ cyan. CdSe/CdS NR hydrogels were printed layer by layer directly after each other without any drying time. After samples with different numbers of printing cycles were printed $(5,25,100,400)$, the printed substrates were placed in a chamber with a humidity of $75 \%$. The initialization of the gelation was started by placing the chamber for $1 \mathrm{~min}$ at $80{ }^{\circ} \mathrm{C}$ in an oven. Afterward, the CdSe/CdS NR hydrogels were dried slowly in the chamber to form films of CdSe/CdS NR xerogel networks. 
Spectroscopic Characterization. The UV/vis absorption spectra of the CdSe/CdS NR solutions and the CdSe/CdS NR gel electrodes were recorded with an Agilent Cary 5000 absorption spectrophotometer equipped with an Agilent DRA-2500 integrating sphere. Emission spectra and photoluminescence lifetime measurements of the solution and the substrates were recorded using a Fluoromax-4 spectrometer from Horiba. The photoluminescence quantum yield (PLQY) was measured in an absolute mode using a DUAL-FL spectrometer from Horiba equipped with a Quanta-Phi integrating sphere.

Scanning Electron Microscopy (SEM). The morphology and thickness of the gel layers at the electrodes were investigated via scanning electron microscopy. A JEOL JSM 6700F microscope, which was equipped with a cold field emission gun as the radiation source, was used. The glass substrates were fixed with carbon badges on brass holders connected using silver conductive paint to increase the conductivity.

Transmission Electron Microscopy (TEM). The structures of the xerogels and rehydrated gels were examined by transmission electron microscopy. All measurements were carried out on an FEI Tecnai G2 F20 microscope equipped with a $200 \mathrm{kV}$ field emission gun. Hydrogel and rehydrated xerogel samples were prepared by drop-casting a small volume of the solution containing gel fragments onto carbon-coated copper grids (Quantifoil, $300 \mathrm{mesh}$ ). The xerogel sample was prepared by gently pressing the grid onto the gel.

Atomic Absorption Spectroscopy (AAS). The Cd concentration of the aqueous NR solution was determined via atomic absorption spectroscopy (AAS) using a Varian AA140 instrument equipped with an air/acetylene (1.5:3.5) flame atomizer. The samples were prepared by dissolving dried toluene-based solutions of the nanoparticles in aqua regia. Aqua regia was diluted in $50 \mathrm{~mL}$ measurement cylinders with deionized water. Five standard $\mathrm{Cd}^{2+}$ solutions were measured to obtain a calibration line.

Electrochemical Measurements. The applied electrochemical setup was based on a ModuLab XM ECS potentiostat from Solartron. All shown measurements were realized using a three-electrode setup based on the prepared samples as working electrodes, $\mathrm{Ag} / \mathrm{AgCl}$ reference electrodes purchased from $\mathrm{BASi}$, and platinum wires as counter electrodes. The electrodes were placed in a polytetrafluoroethylene measurement cell with an illumination window of $0.2 \mathrm{~cm}^{2}$. The same geometric area of the electrode was in contact with the electrolyte. In the case of all measurements, an aqueous solution of 0.5 $\mathrm{M} \mathrm{Na}_{2} \mathrm{SO}_{3}$ with a $\mathrm{pH}$ value of 9.6 was applied as the electrolyte. $\mathrm{Na}_{2} \mathrm{SO}_{3}$ is an effective hole catcher in the case of metal-chalcogenide materials. $^{29,30}$

In the case of IMPS and LSV measurements, light-emitting diodes (LEDs) were applied. The wavelength-resolved photon fluxes of these LEDs with the wavelengths $468,525,594$, and $615 \mathrm{~nm}$ are shown in the Supporting Information (SI Figure S1).

Measurement of Intensity-Modulated Photocurrent Spectra. The utilization of a 7270 dual reference lock-in amplifier purchased from Signal Recovery made an effective recording of the IMPS spectra possible. A scheme of such a setup is given in different literature sources. ${ }^{30}$ The photon flux of all as light source applied modulated LEDs is in good approximation frequency independent in the frequency domain, which is applied in the experiments (SI Figure S2). The obtained data was evaluated with the software Acquire, which is a product of the same company as the lock-in amplifier.

Measurement of External Photocurrent Efficiency Spectra. A spectrum of the monochromator Xe-lamp setup, which was applied to record the external photocurrent efficiency spectra, can be seen in SI Figure S3. The shown spectrum was measured with a $\mathrm{Si}$ photodiode of the type FDS100 from Thorlabs and applied as a reference spectrum to obtain the external photocurrent efficiency from the measured photocurrent. The lamp and the Czerny-Turner monochromator of the type MSH 300 were supplied by LOTQuantumDesign. All spectra were recorded under modulation of the light beam by a C-995 optical chopper purchased from Laser 2000.

Measurement of Linear Sweep Voltammograms. LSVs were obtained under a bias potential sweep from -0.65 to $0.3 \mathrm{~V}$ vs $\mathrm{Ag} /$
$\mathrm{AgCl}$ with a sweeping velocity of $4 \mathrm{mV} / \mathrm{s}$. The square-wave pulsed illumination of $40 \mathrm{mHz}$ frequency was obtained using a $468 \mathrm{~nm}$ LED. The $12.5 \mathrm{~V}$ pulses were generated by a Hameg HMF 2525 frequency generator. The photon flux of the LED in the case of the LSVs was 14 $\mathrm{nmol} / \mathrm{s}$. In the case of long-term measurements, the measurement cell and sample were never moved during the whole experiment. The illumination of the measurement sample was prevented between two LSV measurements. The electrodes were kept during the whole experimental period in the measurement cell, which was stored in a dark faradic cage.

\section{RESULTS AND DISCUSSION}

Structural and Spectroscopic Analyses. This work shows why the long-term behavior of rehydrated $\mathrm{CdSe} / \mathrm{CdS}$ NR-based gel-network-coated electrodes is strongly dependent on whether an electrode is printed or gelated in a cuvette. Gelated and nongelated CdSe/CdS NR-coated electrode interfaces were produced by adjusting the number of printing cycles from 5 to 400 . It is necessary to mention that a printing cycle is not equivalent to a discrete geometrical layer. An overview of the produced and studied electrode samples is given in the Supporting Information (SI Table S1).

The morphology and thickness of the printed $\mathrm{CdSe} / \mathrm{CdS}$ NR xerogel-coated electrodes and cuvette-gelated xerogelcoated electrodes were investigated by top-view and cross section SEM measurements (Figure 1). As already published in

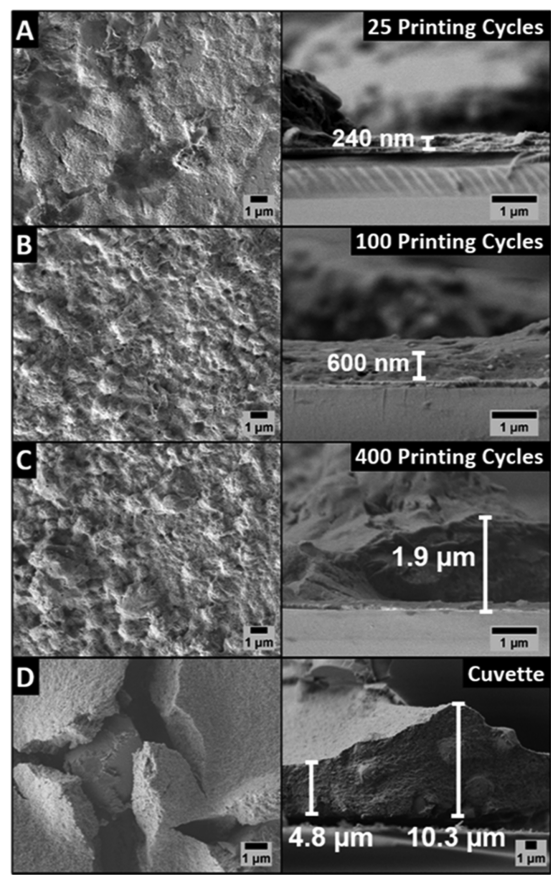

Figure 1. SEM images of the gel surfaces (left side) and images of the cross sections of the same gels (right side) of CdSe/CdS NR of (A) 25 printed cycles, (B) 100 printed cycles, (C) 400 printed cycles, and (D) a network gelated in a cuvette.

our previous work, the printed area becomes more compact and the thickness increases with increasing printing cycles. ${ }^{15,23}$ The main difference between the xerogel-coated electrodes produced via 100 or 400 printing cycles (Figure $1 \mathrm{~B}, \mathrm{C}$ ) and the samples obtained in a cuvette (Figure 1D) is the appearance of distinct cracks on the nanoparticle film surface in the latter case. Structural cracks can be ascribed to the drying. Gels of the cuvette origin are less homogeneous in their thickness 
(Figure 1D, right), since these gels are products of a rapid gelation process, which starts at multiple randomly ordered centers, so the interconnectivity of such gel networks is quite inhomogeneous. Such an inhomogeneity leads to an inconsistent drying and, during this process, the rupture of the structure. This process is in the case of printed gels compensated by some sort of a "crack healing" process, since nanoparticles of later printing cycles can fill cracks or empty spaces in the layers of earlier printing cycles. This mechanism leads to a more homogeneous gel. The optical absorption of the samples as well as the number of absorbed photons under excitation is increasing with the sample thickness (SI Figure S4).

In the UV/vis absorption spectrum of a CdSe/CdS NR solution (Figure 2, black line), the absorption at wavelengths

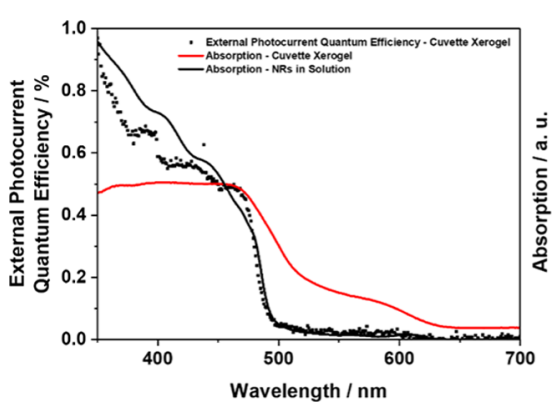

Figure 2. Absorption spectra of a solution of $100 \mu \mathrm{L}$ of CdSe/CdS NRs in $3 \mathrm{~mL}$ of toluene solution (black line) with an absorption of $15.8 \%$ at $463 \mathrm{~nm}$ and of an electrode prepared via gelation in a cuvette (red line) with an absorption of $86.4 \%$ at $463 \mathrm{~nm}$ are compared to the external photocurrent efficiency spectra of the electrode (black dots). It is obvious that the external photocurrent quantum efficiency of an electrode matches better the absorption of the solution than its own. This proves a strong limitation of the penetration depth of photons in the semiconductor gel network.

above $500 \mathrm{~nm}$ can be assigned to the absorption of the CdSe core, whereas the rise of the absorption below $500 \mathrm{~nm}$ is connected to the band edge of $\mathrm{CdS}$, with a first local absorption maximum at $463 \mathrm{~nm}$. In the case of a xerogel of the cuvette origin, the absorption onsets corresponding to the different NR parts become more prominent.
The slope of a photocurrent spectrum of a gel of the cuvette origin (Figure 2, black dots) is more similar to that of the absorption spectrum of a diluted NR solution than to those of its own absorption spectra. This circumstance is explainable with the limited illumination depth. The presented photocurrent spectrum is based on the illumination of only the first NR layers next to the ITO glass. Slight differences of both spectra below $350 \mathrm{~nm}$ can be attributed to optical effects like scattering at boundaries, as in the case of a monolayer of NRs at the electrode, the absorption and photocurrent spectra would match. ${ }^{31}$

Basic Photoelectrochemical Measurements. The main aim of our electrochemical analysis of porous semiconductor nanoparticle-based xerogel structures is to reveal the connection between the electric properties of the material and its structural organization and band structure. In Figure $3 \mathrm{~A}$, a schematic illustration of the applied photoelectrochemical cell and the photo-oxidation of the sulfite occurring during the illumination is shown. The band structure of the semiconductor nanorods is dominated by the nearly similar energetic positions of the conduction bands of the CdS shell and the CdSe seed, which enables electron transport across the whole gel network, while the holes are trapped in the CdSe seed region (Figure 3B). ${ }^{13,14,32}$ It should be mentioned that even if the CdSe core is placed inside the CdS nanorod a tunneling of electrons from the electrolyte species to trapped holes is possible. The applied synthesis procedure creates a $\mathrm{CdS}$ shell around the core with a thickness of less than $0.5 \mathrm{~nm}$. Such a distance makes the tunneling of charges possible so that holes located in the CdSe core can be addressed by different electrolyte species. ${ }^{10,33,34}$

Linear sweep voltammograms (LSVs) of the electrodes were recorded under on-off illumination of the systems (Figure 4, SI Figure S5). Xerogels based on 25 printing cycles show a slightly negative photocurrent below $-0.3 \mathrm{~V}$ (Figure 4A). This photocurrent is connected to the reduction of protons or water molecules, ${ }^{35,36}$ which is mainly happening at the first layer of nanorods directly connected to ITO. The layer of nanorods directly connected to the substrate via MPTMS has the highest intrinsic photocurrent quantum efficiency of all particles in the gel sample. This is explainable with the related mechanism of the charge transport, which requires the least number of
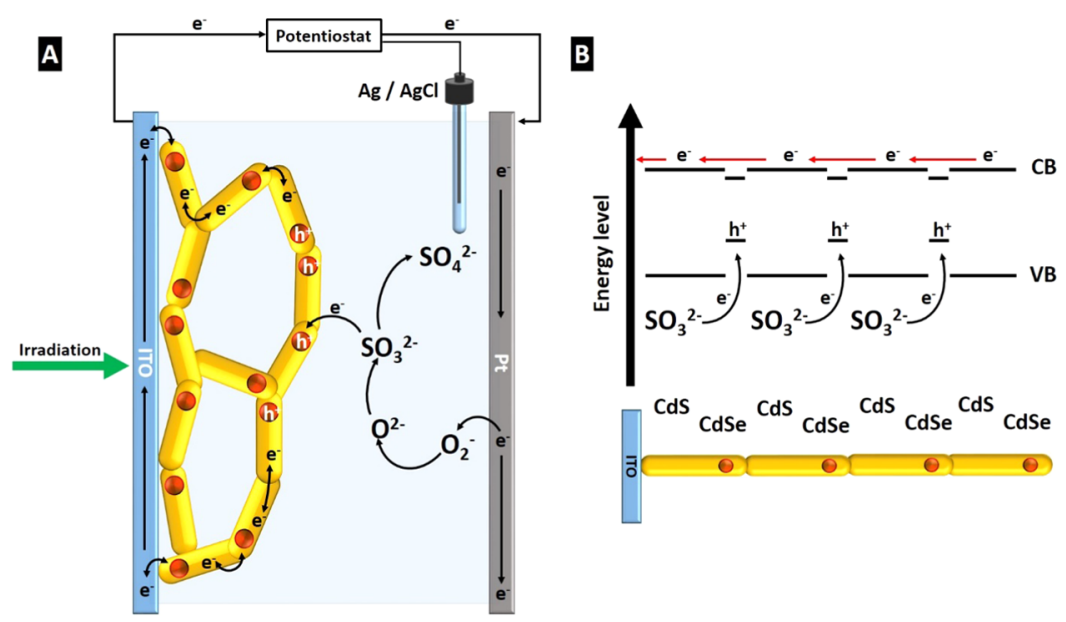

Figure 3. (A) Scheme of the photoelectrochemical photo-oxidation of the sulfite during illumination. Unless otherwise indicated, the nanoparticle network is illuminated through the ITO electrode. (B) Scheme of the band structure of CdSe/CdS under illumination, which leads to the trapping of holes in the valence band of the CdSe seed and the delocalization of electrons across the particle network. 




Figure 4. Linear sweep voltammograms of gels printed with (A) 25, (B) 100, and (C) 400 cycles and (D) NR networks gelated in a cuvette. The LSVs were measured under an on-off illumination with the periodic time of $25 \mathrm{~s}$ using a $468 \mathrm{~nm}$ LED at the presented bias potentials.

steps. ${ }^{37}$ This first category of NRs in the systems provides negative and positive photocurrents at the chosen $\mathrm{pH}$ level. ${ }^{31}$ The generation of negative photocurrent is only hardly possible in the case of NRs, which are connected indirectly to the ITO substrate via linking to other NRs. The second category of particles is nearly exclusively producing positive photocurrent via electron transport to the electrode, since correlated holes are trapped in the CdSe core (Figure 3B). The positive photocurrent generated from the particles of the second category is, in the case of thin semiconductor networks, not high enough to overcome the negative photocurrent from the more effective particle layer next to the substrate (Figure 4A). In contrast, gels produced with 100 printing cycles or more show only positive photocurrent, since the signal of particles of the second category overcomes the negative photocurrent in the investigated area of bias potential completely (Figure 4B-D).

Similar measurements were carried out with printed nongelated electrodes. The samples show the same tendencies and nearly the same absolute photocurrents (SI Figure S5). However, due to differences in the printing process, the total amount of semiconductor NR material is around 30\% lower in the case of gel networks compared to their nongelated NR equivalents with the same number of printing cycles. Considering the lower photon absorption of gels, which yields photocurrents of the same value, we can consider particularly thicker porous networks to be more photoefficient than their nongelated equivalents (SI Table S1). This is explainable with an increased particle-particle interconnection and a lower amount of isolating organic ligands on the surface of the gels. The inexistent particle-particle interconnection in the case of nongelated samples leads to a dissolution process of the electrodes.

Kinetic Measurements of the Photoelectrochemical Process. The proof that the origin of photocurrent is based on a transport process can be provided via IMPS measurements. The application of IMPS as a time-resolved measurement technique is a suitable way to separate distinct photoelectrochemical mechanisms. For a simple submonolayer of NRs, only one semicircle was obtained (SI Figure S6). If more than one semicircle is present, the existence of distinguishable mechanisms of charge carrier transport is presumable. ${ }^{31,38-41}$ The recorded IMPS spectra of gels are a superposition of two semicircles (Figure 5, SI Figures S7 and S8). The rate of the underlying charge carrier transport mechanisms seems to be different by more than 1 order of magnitude, since the semicircles exist at different magnitudes of the frequency range. The semicircle at higher frequencies is related to the charge transport across the particle-electrolyte interface, which is mandatory for the existence of a photocurrent. Contrary to this, a trap-state-related charge transport across the network,

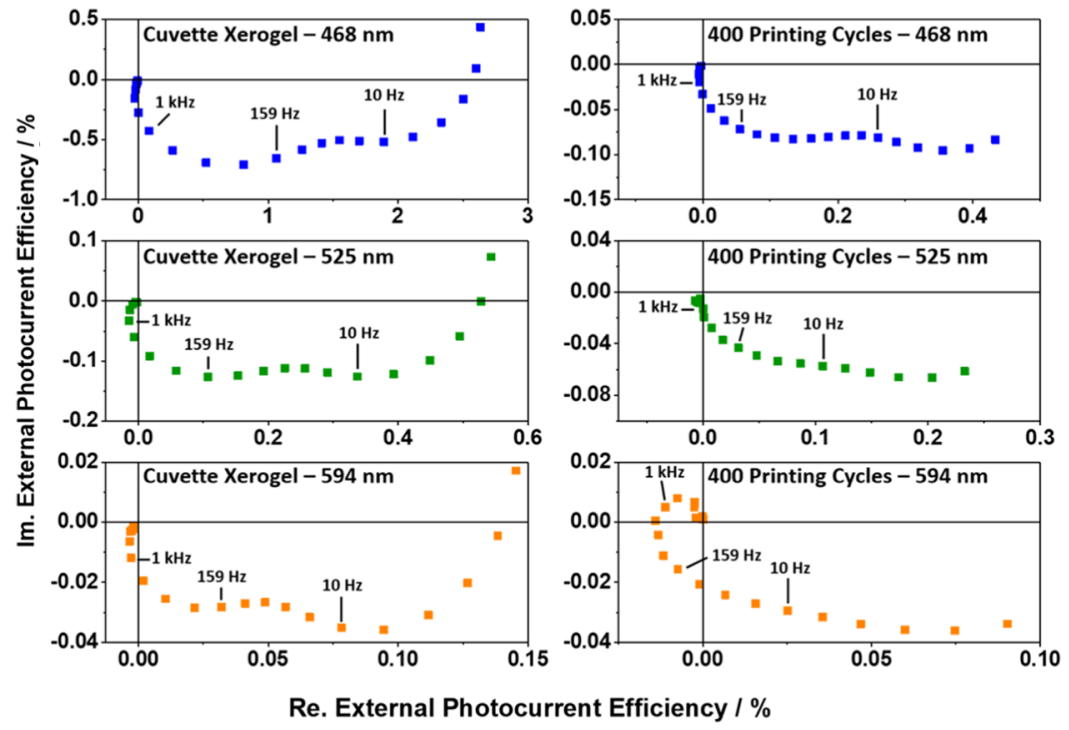

Figure 5. Intensity-modulated photocurrent spectra of (left side) gels of the cuvette origin and (right side) gels based on 400 printing cycles under (blue) $468 \mathrm{~nm}$, (green) $525 \mathrm{~nm}$, and (orange) $594 \mathrm{~nm}$ illumination. It is obvious that the size of the second semicircle increases and becomes dominant with an increase in the excitation wavelength. The excitation frequencies of the samples are given in the spectra. The measurement points at the origin are obtained under an excitation frequency of $10 \mathrm{kHz}$. The lowest applied excitation frequency is $1 \mathrm{~Hz}$ and always connected to the measurement point with the highest photocurrent efficiency. 
which is describable as particle-particle transport, exists at lower frequencies. ${ }^{42}$ The dominance of the particle-electrolyte or the particle-particle transport is expressed by the size of the related semicircles (Figure 5). Since the absolute optical densities of the samples at absorption wavelengths of the CdSe seed and the CdS rod differ remarkably, the penetration depth of photons of different wavelengths in a NR gel varies in magnitude. This circumstance can be applied as the basis for IMPS experiments, which are implemented via an illumination from distinct directions with LEDs of different emission wavelengths (Figure 5 and SI Figure S1). Light of a longer wavelength can penetrate deeper into the xerogel, since it is absorbed only by the CdSe seeds of the NR and not the CdS rod itself (Figure 2). A shift of the illumination wavelength from blue to red causes an excitation of particles more distant from the ITO surface of the electrode. ${ }^{43}$ With an increase in the mean distance between the average excited semiconductor particle and the charge-collecting electrode, the electron transport from the particle to particle becomes the dominating process in the photocurrent generation. This circumstance is illustrated by an increase in the size of the low-frequency semicircle compared to its high-frequency equivalent with the increasing wavelength (Figure 5). The external photocurrent efficiency (EPE) of xerogels prepared in the cuvette is under shorter wavelength excitation $(468 \mathrm{~nm})$ remarkably higher than in the case of the networks obtained by 400 printing cycles (Figure 5). By increasing the excitation wavelength (525 or $594 \mathrm{~nm}$ ), the EPE of printed xerogels and xerogels gelated in a cuvette equalizes. The density of particles in combination with a better charge transport in the printed particle networks could explain the dominance of the semicircle at low frequencies in the case of printed gels.

Long-Term Studies of the Photoelectrochemical Process. During a set of LSV measurements on one sample, in which the single measurement was repeated every couple of minutes, the noticeable photocurrent increased significantly from measurement to measurement (Figure 6).

The presence of hydration as the main factor of activation explains the long-term evolution of the photocurrent in the case of gels (Figures 6 and 7).

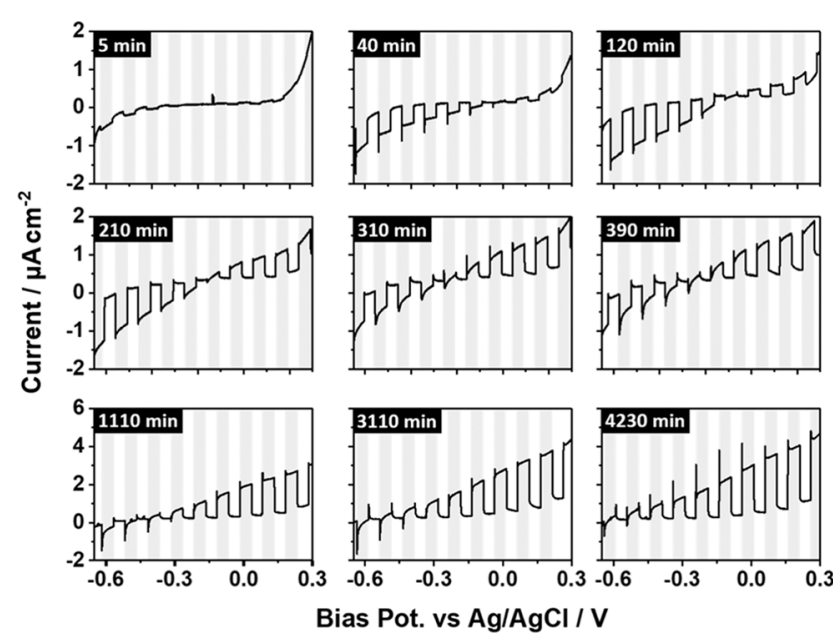

Figure 6. LSV measurements of a 400-printing-cycle gel at different stages of the mentioned hydration. A shift of the point at which positive and negative photocurrents are equal to more negative potentials is evident.
As an example, the photocurrent evolution of a 400-printingcycle-based CdSe/CdS NR xerogel-coated electrode is shown over a time period of 14 days (Figures 6 and 8). Over the whole investigated time scale, a significant increase of the photocurrent is observable. This phenomenon existed in the case of all samples studied (Figures 9and 10). The corresponding IMPS spectra (Figure 8, inset) show a small semicircle at lower frequencies and a larger semicircle at higher frequencies at all investigated times. Both semicircles grow over the investigated time, with the lower frequency semicircle growing comparably stronger. As mentioned before, this semicircle is based on the transport process of charges through the gel network. It is reasonable that, in particular, this process is amplified by the hydration of the gel.

Besides the increase in the intensity of the photocurrent at positive bias potentials, another effect is noticeable. A remarkable shift of the bias potential at which positive and negative photocurrents are equal can be perceived (Figure 10). This second phenomenon is associated with the increase in the percentage of photocurrent generated by the second category of NRs, which is more distant to the ITO substrate. Accordingly, the recorded data shows the interconnection between charge carrier transport within the xerogel network, the rehydration process of the xerogel structure, and the photocurrent direction. One century ago, Pascheles and Ostwald considered the hydration of gels as an important research subject. ${ }^{44,45}$ Hydration kinetics of classical gels like gelatine was already studied in relation to factors like electrolyte type, temperature, and $\mathrm{pH}$ level. ${ }^{4,45}$ Over time, the swelling of polymer-based gels attracted the scientific interest. Examples of polymer gels containing semiconducting particles are known, which gave them sensing functionality or even enabled the swelling mechanism. ${ }^{46,47}$ Apart from those known mechanisms, information about the hydration kinetics of mechanical self-supporting semiconductor nanoparticlebased gel networks is not available. Nevertheless, it is easy to model the kinetic process, since the fundamental mechanisms of hydration are based on the interaction between the electrolyte and solid network. A fundamental step of the generation of positive photocurrents is the adsorption of sulfite molecules at the nanoparticle surface sites, which are next to trapped holes. ${ }^{48}$ The total amount of electrolyte molecules in the system is much higher than the number of nanoparticles or more specifically their addressable surface states. This means that during a hydration the amount of electrolyte molecules in solution stays quasiconstant and the number of addressable surface states increases until the full state of hydration is reached. This fact leads to the description of hydration as a pseudo-first-order kinetic reaction (eq 1). ${ }^{44}$ The equation describes the time dependence of the amplitude of the photocurrent $I_{\text {photo }}$ in relation to the maximum photocurrent $I_{\max }$ that can be reached. The hydration reaction of the gel is described via a kinetical constant $k$.

$$
I_{\text {photo }}=I_{\max }-I_{\max } \cdot \mathrm{e}^{(-k \cdot t)}
$$

A description of xerogels with the presented kinetic equation is possible, with the exception of the ones of the cuvette origin (Figure 10). Hydration processes across periods of several days are recognizable only in the case of printed samples, which do not show cracks in the NR film (Figures 9 and 10). The hydration velocity of porous semiconductor nanoparticle-based 

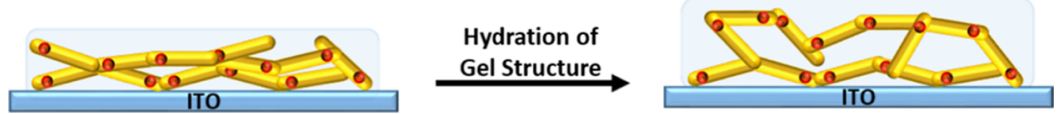

Figure 7. General mechanism of hydration, which is caused by the rehydration of the network structure. The shown rehydration process can also be monitored by transmission electron microscopy (TEM, SI Figure S10).

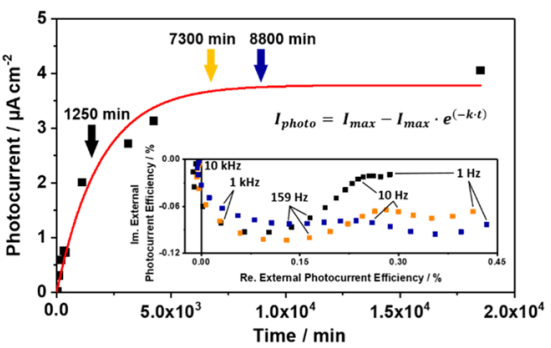

Figure 8. Photocurrent of a gel electrode prepared via 400 printing cycles in relation to its hydration time. The evolution of the current is fitted with a first-order kinetics equation, which is presented in the figure. The hydration process takes place over a period of days. IMPS spectra of the system were recorded at different stages of the rehydration process. The IMPS measurements took place after (black arrow and dots) 1250, (yellow arrow and dots) 7300, and (blue arrow and dots) $8800 \mathrm{~min}$

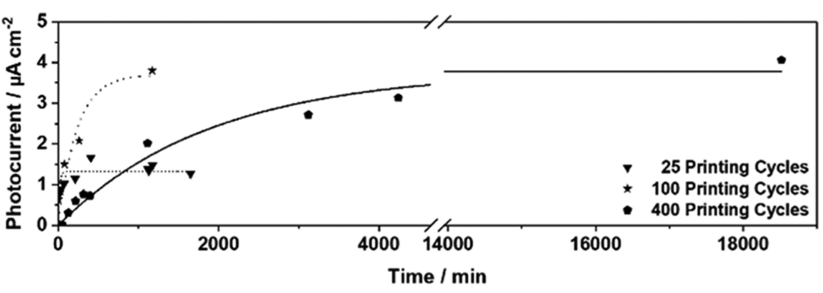

Figure 9. Time-dependent evolution of the measured photocurrent presented for 25 printing cycles (triangles), 100 printing cycles (stars), and 400 printing cycles (pentagons) of gels. The first-order reaction kinetics is related to the underlying reaction mechanism (Figures 3B and 7).

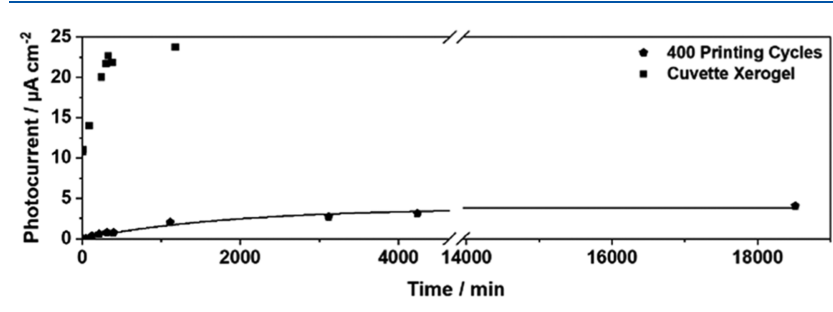

Figure 10. Time-dependent increase of the photocurrent efficiencies presented for the case of (pentagons) 400 printed cycles and (circles) a gel from the cuvette origin. The difference in the velocity of the photocurrent increase is related to the extremely different hydration mechanisms of both samples.

networks was observed to be related to the thickness of the swelling samples (Figure 9).

Since thinner layers are faster hydrated, an increase in the electrochemical activation is recognizable for gelated and nongelated samples (Figures 9 and 10, SI Figure S9). The rate constants obtained by fitting the data points can be found in the Supporting Information (SI Table S2). In contrast to the slow hydration of printed samples, networks obtained from gelation in cuvettes show a hydration within minutes (Figure 10).
This difference of at least 2 orders of magnitudes in the time scale of hydration can be explained with the structure of gels with the cuvette origin. The structural cracks in these gels play the role of hydration notches. A fast hydration of a gel interface is much quicker through these "fast lanes" of electrolyte diffusion. An overview of the short- and long-time kinetic investigations leads to the conclusion that a hydration process, which has the duration of days and weeks, is necessary to activate the printed xerogel electrodes. The activation is mandatory to have a reproducible photoelectrochemical performance of the electrodes.

\section{CONCLUSIONS}

A comparison of nongelated and gelated electrodes shows an increase in the intrinsic photocurrent efficiency, which can be explained with a NR surface activation via gelation. According to our model, the connection of the CdSe/CdS NRs within the gel network leads to a delocalization of the electron over larger parts of the gel structure, while the holes remain in the CdSe core regions. By investigating the photocurrent obtained under illumination in relation to the time after the start of hydration by means of LSV, it was possible to propose a mechanism of charge carrier transport. The enhanced transport is connected to the hydration mechanism of the gel. IMPS measurements showed that with progressing hydration the transport mechanism of charge carriers becomes dominant in relation to the interface transfer mechanism of charge carriers. This causes a shift in the equilibrium of positive and negative photocurrents. The shift leads to a higher overall current in the investigated electrochemical potential window. The suppression of one current direction causes a phenomenological rectifying character of the system. This behavior is classically known for diodes. To the best of our knowledge, our contributed work is the first paper that describes this effect. In summary, the application of a quasi-type-II heterostructure in gel-type assemblies was found to be an extremely beneficial factor and, further, materials with quasi-type-II structure like $\mathrm{CdTe} / \mathrm{ZnS},{ }^{49} \mathrm{InP} / \mathrm{CdS},{ }^{50} \mathrm{ZnSe} / \mathrm{CdS},{ }^{51}$ and $\mathrm{Ag}_{2} \mathrm{~S} / \mathrm{AgInS}_{2}{ }^{52}$ should be tested regarding their charge carrier transfer properties. The observed performance of the different samples displays the requirement to optimize the printing layout of the gel electrodes. It is obvious that the cracks in the coating played a key role as gateways for the hydration and therefore enable the activation of the electrode. The existence of these gaps seems to amplify the hydration velocity by at least 2 orders of magnitude. Future printed systems should be designed in such a way that a pattern of hydration channels exists on purpose.

\section{ASSOCIATED CONTENT}

\section{(s) Supporting Information}

The Supporting Information is available free of charge at https://pubs.acs.org/doi/10.1021/acs.langmuir.9b03708.

Parameters of the spectroelectrochemical setup; further optical and spectroelectrochemical characterization of 
the applied photoelectrodes; and TEM images of the dried and rehydrated gels (PDF)

\section{AUTHOR INFORMATION}

\section{Corresponding Author}

Nadja C. Bigall - Institute of Physical Chemistry and Electrochemistry and Laboratory of Nano and Quantum Engineering, Leibniz Universität Hannover, D-30167 Hannover, Germany; Cluster of Excellence PhoenixD, (Photonics, Optics, and Engineering-Innovation Across Disciplines), 30167 Hannover, Germany; ๑ orcid.org/00000003-0171-1106; Email: nadja.bigall@pci.uni-hannover.de

\section{Authors}

Jan F. Miethe - Institute of Physical Chemistry and Electrochemistry and Laboratory of Nano and Quantum Engineering, Leibniz Universität Hannover, D-30167 Hannover, Germany

Franziska Luebkemann - Institute of Physical Chemistry and Electrochemistry and Laboratory of Nano and Quantum Engineering, Leibniz Universität Hannover, D-30167 Hannover, Germany

Anja Schlosser - Institute of Physical Chemistry and Electrochemistry and Laboratory of Nano and Quantum Engineering, Leibniz Universität Hannover, D-30167 Hannover, Germany

Dirk Dorfs - Institute of Physical Chemistry and Electrochemistry and Laboratory of Nano and Quantum Engineering, Leibniz Universität Hannover, D-30167 Hannover, Germany; Cluster of Excellence PhoenixD, (Photonics, Optics, and Engineering-Innovation Across Disciplines), 30167 Hannover, Germany; @ orcid.org/00000001-6175-4891

Complete contact information is available at: https://pubs.acs.org/10.1021/acs.langmuir.9b03708

\section{Notes}

The authors declare no competing financial interest.

\section{ACKNOWLEDGMENTS}

The authors are grateful for financial support from the German Federal Ministry of Education and Research (BMBF) within the framework of the program NanoMatFutur, support code 03X5525, and the European Research Council (European Union's Horizon 2020 research and innovation program, grant agreement 714429). Furthermore, the authors would like to acknowledge the Laboratory of Nano and Quantum Engineering (LNQE) at the Leibniz Universität Hannover for support. D.D. and F.L. thank the Volkswagen Foundation (Lower Saxony/Israel cooperation, Grant ZN2916), and D.D. thanks the DFG (research grant 1580/5-1). N.C.B. wants to thank the DFG (research grant BI 1708/4-1) for funding. A.S. thanks the Hannover School for Nanotechnology (hsn) for funding. The project has in part been funded by the Deutsche Forschungsgemeinschaft (DFG, German Research Foundation) under Germany's Excellence Strategy within the Cluster of Excellence PhoenixD (EXC 2122, Project ID 390833453). The authors furthermore acknowledge Armin Feldhoff as well as Jürgen Caro for providing the SEM facilities. In addition, we would like to thank P. Mühr, M. Köhler, J. Kuckuck, and W. Becker for their valuable help in realizing the electrochemical setup.

\section{REFERENCES}

(1) Peng, Z. A.; Peng, X. Formation of High-Quality CdTe, CdSe, and $\mathrm{CdS}$ Nanocrystals Using $\mathrm{CdO}$ as Precursor. J. Am. Chem. Soc. 2001, 123, 183-184.

(2) Murray, C. B.; Norris, D. J.; Bawendi, M. G. Synthesis and Characterization of Nearly Monodispers $\mathrm{CdE}(\mathrm{E}=\mathrm{S}$, Se, Te $)$ Semiconductor Nanocrystallites. J. Am. Chem. Soc. 1993, 115, 87068715.

(3) Spanhel, Y.; Haase, M.; Weller, H.; Hengleiir, A. Photochemistry of Colloidal Semiconductors. 20. Surface Modification and Stability of Strong Luminescing CdS Particles. J. Am. Chem. Soc. 1987, 109, 5649-5655.

(4) Gaponik, N.; Talapin, D. V.; Rogach, A. L.; Hoppe, K.; Shevchenko, E. V.; Kornowski, A.; Eychmüller, A.; Weller, H. ThiolCapping of C,dTe Nanocrystals: An Alternative to Organometallic Synthetic Routes. J. Phys. Chem. B 2002, 106, 7177-7185.

(5) Katari, J. E. B.; Colvin, V. L.; Alivisatos, A. P. X-Ray Photoelectron Spectroscopy of CdSe Nanocrystals with Applications to Studies of the Nanocrystal Surface. J. Phys. Chem. A 1994, 98, 4109-4117.

(6) Talapin, D. V.; Rogach, A. L.; Mekis, I.; Haubold, S.; Kornowski, A.; Haase, M.; Weller, H. Synthesis and Surface Modification of Amino-Stabilized CdSe, CdTe and InP Nanocrystals. Colloids Surf., A 2002, 202, 145-154.

(7) Li, L.; Protière, M.; Reiss, P. Economic Synthesis of High Quality InP Nanocrystals Using Calcium Phosphide as the Phosphorus Precursor. Chem. Mater. 2008, 20, 2621-2623.

(8) Malik, M. A.; O’Brien, P.; Norager, S.; Smith, J. Gallium Arsenide Nanoparticles: Synthesis and Characterisation. J. Mater. Chem. 2003, 13, 2591-2595.

(9) Dick, K. A.; Deppert, K.; Larsson, M. W.; Mårtensson, T.; Seifert, W.; Wallenberg, L. R.; Samuelson, L. Synthesis of Branched "nanotrees" by Controlled Seeding of Multiple Branching Events. Nat. Mater. 2004, 3, 380-384.

(10) Carbone, L.; Nobile, C.; De Giorgi, M.; Della Sala, F.; Morello, G.; Pompa, P.; Hytch, M.; Snoeck, E.; Fiore, A.; Franchini, I. R.; Nadasan, M.; Silvestre, A. F.; Chiodo, L.; Kudera, S.; Cingolani, R.; Krahne, R.; Manna, L. Synthesis and Micrometer-Scale Assembly of Colloidal CdSe/CdS Nanorods Prepared by a Seeded Growth Approach. Nano Lett. 2007, 7, 2942-2950.

(11) Tessier, M. D.; Spinicelli, P.; Dupont, D.; Patriarche, G.; Ithurria, S.; Dubertret, B. Efficient Exciton Concentrators Built from Colloidal Core/Crown CdSe/CdS Semiconductor Nanoplatelets. Nano Lett. 2014, 14, 207-213.

(12) Alemán, J. V.; Chadwick, A. V.; He, J.; Hess, M.; Horie, K.; Jones, R. G.; Meisel, I.; Mita, I.; Moad, G.; Penczek, S.; Stepto, R. F. T. Definitions of Terms Relating to the Structure and Processing of Sols, Gels, Networks, and Inorganic - Organic Hybrid Materials. Pure Appl. Chem. 2007, 79, 1801-1829.

(13) Kunneman, L. T.; Zanella, M.; Manna, L.; Siebbeles, L. D. A.; Schins, J. M. Mobility and Spatial Distribution of Photoexcited Electrons in CdSe/CdS Nanorods. J. Phys. Chem. C 2013, 117, 31463151.

(14) Eshet, H.; Grünwald, M.; Rabani, E. The Electronic Structure of CdSe/CdS Core/Shell Seeded Nanorods: Type-I or Quasi-TypeII? Nano Lett. 2013, 13, 5880-5885.

(15) Lübkemann, F.; Miethe, J. F.; Steinbach, F.; Rusch, P.; Schlosser, A.; Zámbó, D.; Heinemeyer, T.; Natke, D.; Zok, D.; Dorfs, D.; Bigall, N. C. Patterning of Nanoparticle-Based Aerogels and Xerogels by Inkjet Printing. Small 2019, 15, No. 1902186.

(16) Yu, H.; Brock, S. L. Effects of of Nanoparticle Shape on the Morphology and Properties of Porous CdSe Assemblies (Aerogels). ACS Nano 2008, 1, 316-319.

(17) Sánchez-Paradinas, S.; Dorfs, D.; Friebe, S.; Freytag, A.; Wolf, A.; Bigall, N. C. Aerogels from CdSe/CdS Nanorods with Ultra-Long Exciton Lifetimes and High Fluorescence Quantum Yields. Adv. Mater. 2015, 27, 6152-6156.

(18) Rusch, P.; Niemeyer, F.; Pluta, D.; Schremmer, B.; Lübkemann, F.; Rosebrock, M.; Schäfer, M.; Jahns, M.; Behrens, P.; Bigall, N. C. 
Versatile Route to Core-Shell Reinforced Network Nanostructures. Nanoscale 2019, 11, 15270-15278.

(19) Schlosser, A.; Meyer, L. C.; Lübkemann, F.; Miethe, J. F.; Bigall, N. C. Nanoplatelet Cryoaerogels with Potential Application in Photoelectrochemical Sensing. Phys. Chem. Chem. Phys. 2019, 21, 9002-9012.

(20) Freytag, A.; Colombo, M.; Bigall, N. C. Catalytic Properties of Cryogelated Noble Metal Aerogels. Z. Physiol. Chem. 2016, 231, 6375.

(21) Hendel, T.; Lesnyak, V.; Kühn, L.; Herrmann, A. K.; Bigall, N. C.; Borchardt, L.; Kaskel, S.; Gaponik, N.; Eychmüller, A. Mixed Aerogels from Au and CdTe Nanoparticles. Adv. Funct. Mater. 2013, 23, 1903-1911.

(22) Berestok, T.; Guardia, P.; Estradé, S.; Llorca, J.; Peiró, F.; Cabot, A.; Brock, S. L. CuGaS2 and $\mathrm{CuGaS2}-\mathrm{ZnS}$ Porous Layers from Solution-Processed Nanocrystals. Nanomaterials 2018, 8, 1-14.

(23) Lübkemann, F.; Anselmann, R.; Kodanek, T.; Bigall, N. C. Inkjet Printing of Aqueous Photoluminescent CdSe/CdS Nanorods on Solid Substrates. Chem. Ing. Tech. 2017, 89, 807-813.

(24) Halivni, S.; Shemesh, S.; Waiskopf, N.; Vinetsky, Y.; Magdassi, S.; Banin, U. Inkjet Printed Fluorescent Nanorod Layers Exhibit Superior Optical Performance over Quantum Dots. Nanoscale 2015, 7, 19193-19200.

(25) Böberl, M.; Kovalenko, M. V.; Gamerith, S.; List, E. J. W.; Heiss, W. Inkjet-Printed Nanocrystal Photodetectors Operating up to 3 Mm Wavelengths. Adv. Mater. 2007, 19, 3574-3578.

(26) Yu, W. W.; Qu, L.; Guo, W.; Peng, X. Experimental Determination of the Extinction Coefficient of $\mathrm{CdTe}, \mathrm{CdSe}$, and CdS Nanocrystals. Chem. Mater. 2003, 15, 2854-2860.

(27) Kodanek, T.; Banbela, H. M.; Naskar, S.; Adel, P.; Bigall, N. C.; Dorfs, D. Phase Transfer of 1- and 2-Dimensional Cd-Based Nanocrystals. Nanoscale 2015, 7, 19300-19309.

(28) Kern, W.; Puotinen, D. A. Cleaning Solutions Based on Hydrogen Peroxide for Use in Silicon Semiconductor Technology. RCA Rev. 1970, 31, 187-206.

(29) Vaneski, A.; Schneider, J.; Susha, A. S.; Rogach, A. L. Colloidal Hybrid Heterostructures Based on II-VI Semiconductor Nanocrystals for Photocatalytic Hydrogen Generation. J. Photochem. Photobiol., C 2014, 19, 52-61.

(30) Hickey, S. G.; Riley, D. J. Photoelectrochemical Studies of CdS Nanoparticle-Modified Electrodes. J. Phys. Chem. B 1999, 103, 45994602.

(31) Miethe, J. F.; Lübkemann, F.; Poppe, J.; Steinbach, F.; Dorfs, D.; Bigall, N. C. N. C. Spectroelectrochemical Investigation of the Charge Carrier Kinetics of Gold-Decorated Cadmium Chalcogenide Nanorods. ChemElectroChem 2018, 5, 175-186.

(32) Bridewell, V. L.; Alam, R.; Karwacki, C. J.; Kamat, P. V. CdSe/ CdS Nanorod Photocatalysts: Tuning the Interfacial Charge Transfer Process through Shell Length. Chem. Mater. 2015, 27, 5064-5071.

(33) Coropceanu, I.; Rossinelli, A.; Caram, J. R.; Freyria, F. S.; Bawendi, M. G. Growth of Seeded CdSe/CdS Nanorods with Unity Fluorescence Quantum Yield and Complete Shell to Core Energy Transfer. ACS Nano 2016, 10, 3925-3301.

(34) Kalisman, P.; Nakibli, Y.; Amirav, L. Perfect Photon-toHydrogen Conversion Efficiency. Nano Lett. 2016, 16, 1776-1781.

(35) Berr, M. J.; Vaneski, A.; Mauser, C.; Fischbach, S.; Susha, A. S.; Rogach, A. L.; Jäckel, F.; Feldmann, J. Delayed Photoelectron Transfer in Pt-Decorated CdS Nanorods under Hydrogen Generation Conditions. Small 2012, 8, 291-297.

(36) Berr, M. J.; Wagner, P.; Fischbach, S.; Vaneski, A.; Schneider, J.; Susha, A. S.; Rogach, A. L.; Jäckel, F.; Feldmann, J. Hole Scavenger Redox Potentials Determine Quantum Efficiency and Stability of PtDecorated CdS Nanorods for Photocatalytic Hydrogen Generation. Appl. Phys. Lett. 2012, 100, No. 223903.

(37) Miethe, J. F.; Lübkemann, F.; Bigall, N. C.; Dorfs, D. Photoluminescence Lifetime Based Investigations of Linker Mediated Electronic Connectivity Between Substrate and Nanoparticle. Front. Chem. 2019, 7, 207.
(38) Miethe, J. F.; Schlosser, A.; Eckert, J. G.; Lübkemann, F.; Bigall, N. C. Electronic Transport in CdSe Nanoplatelet Based Polymer Fibres. J. Mater. Chem. C 2018, 6, 10916-10923.

(39) Wang, H.; Gonzalez-Pedro, V.; Kubo, T.; Fabregat-Santiago, F.; Bisquert, J.; Sanehira, Y.; Nakazaki, J.; Segawa, H. Enhanced Carrier Transport Distance in Colloidal PbS Quantum-Dot-Based Solar Cells Using ZnO Nanowires. J. Phys. Chem. C 2015, 119, 27265-27274.

(40) de Jongh, P. E.; Vanmaekelbergh, D. Investigation of the Electronic Transport Properties of Nanocrystalline Particulate TiO2 Electrodes by Intensity-Modulated Photocurrent Spectroscopy. J. Phys. Chem. B. 1997, 101, 2716-2722.

(41) Mao, X.; Zhou, R.; Zhang, S.; Ding, L.; Wan, L.; Qin, S.; Chen, Z.; Xu, J.; Miao, S. High Efficiency Dye-Sensitized Solar Cells Constructed with Composites of $\mathrm{TiO} 2$ and the Hot-Bubbling Synthesized Ultra-Small SnO2 Nanocrystals. Sci. Rep. 2016, 6, No. 19390.

(42) Guillén, E.; Ramos, F. J.; Anta, J. A.; Ahmad, S. Elucidating Transport-Recombination Mechanisms in Perovskite Solar Cells by Small-Perturbation Techniques. J. Phys. Chem. C 2014, 118, 2291322922.

(43) Hodes, G.; Howell, I. D. J.; Peter, L. M. Nanocrystalline Photoelectrochemical Cells A New Concept In Photovoltaic Cells. J. Electrochem. Soc 1992, 139, 3136-3140.

(44) Pascheles, W. Versuche Über Quellung. Pflugers Arch Gesamte Physiol Menschen Tiere 1898, 71, 333-356.

(45) Ostwald, W. Über Den Einfluss von SÄuren Und Alkalien Auf Die Quellung von Gelatine. Pflugers Arch Gesamte Physiol Menschen Tiere 1905, 108, 563-589.

(46) Tatsuma, T.; Takada, K.; Miyazaki, T. UV-Light-Induced Swelling and Visible-Light-Induced Shrinking of a TiO2-Containing Redox Gel. Adv. Mater. 2007, 19, 1249-1251.

(47) Bekiari, V.; Pagonis, K.; Bokias, G.; Lianos, P. Study of Poly(N,N-Dimethylacrylamide)/CdS Nanocomposite Organic/Inorganic Gels. Langmuir 2004, 20, 7972-7975.

(48) Raevskaya, A. E.; Stroyuk, A. L.; Kuchmii, S. Y. CdS Nanoparticle Photocatalysis of the Chain Oxidation of Sulfite Ions by Molecular Oxygen. Theor. Exp. Chem. 2003, 39, 235-241.

(49) Maiti, S.; Debnath, T.; Maity, P.; Ghosh, H. N. Lattice-StrainInduced Slow Electron Cooling Due to Quasi-Type-II Behavior in Type-I CdTe/ZnS Nanocrystals. J. Phys. Chem. C 2015, 119, 84108416.

(50) Wu, K.; Song, N.; Liu, Z.; Zhu, H.; Rodríguez-Córdoba, W.; Lian, T. Interfacial Charge Separation and Recombination in InP and Quasi-Type II InP/CdS Core/Shell Quantum Dot-Molecular Acceptor Complexes. J. Phys. Chem. A 2013, 117, 7561-7570.

(51) Ivanov, S. A.; Piryatinski, A.; Nanda, J.; Tretiak, S.; Zavadil, K. R.; Wallace, W. O.; Werder, D.; Klimov, V. I. Type-II Core/Shell CdS/ZnSe Nanocrystals: Synthesis, Electronic Structures, and Spectroscopic Properties. J. Am. Chem. Soc. 2007, 129, 11708-11719.

(52) Bose, R.; Manna, G.; Jana, S.; Pradhan, N. Ag2S-AgInS2: P-n Junction Heteronanostructures with Quasi Type-II Band Alignment. Chem. Commun. 2014, 50, 3074-3077. 